\title{
Electromagnetic cyclotron instabilities in bi-Kappa distributed plasmas: a quasilinear approach
}

\author{
M. Lazar, ${ }^{1,2, a)}$ P.H. Yoon, ${ }^{3,4, b)}$ and B. Eliasson ${ }^{5, c)}$ \\ 1) Institut für Theoretische Physik, Lehrstuhl IV: Weltraum- und Astrophysik, Ruhr-Universität Bochum, \\ D-44780 Bochum, Germany \\ ${ }^{2)}$ Centre for Mathematical Plasma Astrophysics, Celestijnenlaan 200B, 3001 Leuven, \\ Belgium \\ ${ }^{3)}$ Institute for Physical Science \& Technology, University of Maryland, College Park, \\ $M D 20742$ \\ 4) School of Space Research, Kyung Hee University, Yongin-Si, Gyeonggi-Do, 446-701, \\ Korea \\ 5) SUPA, Physics Department, John Anderson Building, Strathclyde University, Glasgow G4 ONG, Scotland, \\ $U K$
}

(Dated: 15 March 2017)

\begin{abstract}
Anisotropic bi-Kappa distributed plasmas, as encountered in the solar wind and planetary magnetospheres, are susceptible to a variety of kinetic instabilities including the cyclotron instabilities driven by an excess of perpendicular temperature $T_{\perp}>T_{\|}$(where $\|, \perp$ denote directions relative to the mean magnetic field). These instabilities have been extensively investigated in the past, mainly limiting to a linear stability analysis. About their quasilinear (weakly nonlinear) development some insights have been revealed by numerical simulations using PIC and Vlasov solvers. This paper presents a self-consistent analytical approach, which provides for both the electron and proton cyclotron instabilities an extended picture of the quasilinear time evolution of the anisotropic temperatures as well as the wave energy densities.
\end{abstract}

\section{INTRODUCTION}

Electromagnetic wave fluctuations are continuously reported by the observations in space plasmas especially due to the improved capabilities to measure them insitu, e.g., in the solar wind or the more specific planetary magnetospheres. Relevant in this sense are the observational analyses of the magnetic and velocity turbulence provided by Zimbardo et al. (2010) and Bruno and Carbone (2013) for the solar wind and the Earth's magnetosphere, Alexandrova (2008) for the magnetosheath, Cargill et al. (2005) for the cusp regions, Bauer et al. (1995) and Borovsky and Funsten (2003) for the magnetotail, and Lysak (1990) and Stasiewicz et al. (2000) for the ionosphere.

Space plasmas are collisionless and sufficiently homogeneous, such that small-amplitude perturbations can be amplified by the electromagnetic instabilities driven by the kinetic anisotropies of charged particles. In the absence of energetic events, e.g., coronal mass ejections, the deviations from isotropy are small (Štverák et al., 2008; Hellinger et al., 2006) and the growth-rates of the instabilities are expected to be low (e.g., lower than the wavefrequency $\gamma<\omega_{r}$ ). The plasma is initially linearly unstable to electromagnetic instabilities leading to a weak turbulence which can be examined by means of a quasilinear analysis of the Vlasov-Maxwell equations. With a major role in the relaxation of kinetic anisotropies, these

\footnotetext{
a)Electronic mail: mlazar@tp4.rub.de

b) Electronic mail: yoonp@umd.edu

c)Electronic mail: bengt.eliasson@strath.ac.uk
}

instabilities can also be at the origin of enhanced fluctuations observed in the range of cyclotron frequencies of plasma particles. In the solar wind the cyclotron frequency decreases with the distance from the Sun.

In the last decade a systematic effort has been made to understand these instabilities, especially their quasilinear evolution and, implicitly, the effects on particles and plasma properties (Lu, Zhou, and Wang, 2010; Yoon and Seough, 2012; Seough and Yoon, 2012; Yoon et al., 2012; Seough et al., 2013; Eliasson and Lazar, 2015; Sarfraz et al., 2016). Of a particular importance is the cyclotron (resonant) interaction of plasma particles (electrons and protons) with the electromagnetic circularly (or elliptically) polarized wave fields, when the particles satisfy $\omega-k_{\|} v_{\|}=n \Omega_{s}$, where $\omega$ is the wave frequency, $k_{\|}$and $v_{\|}$are the parallel components of the wave-vector and particle velocity, respectively, and $\Omega_{s}=e_{s} B /\left(m_{s} c\right)$ is the nonrelativistic cyclotron frequency for the particle species $s$ ( $s=e$ for electrons, and $s=i$ for ions). By this mechanism the electromagnetic modes undergo either a cyclotron damping in the absence of any free energy of plasma particles, or a cyclotron instability usually driven by an anisotropic temperature $T_{\perp}>T_{\|}$, where $\|, \perp$ denote directions relative to the mean magnetic field. If the cyclotron interaction implies an important fraction of resonant particles, their effects and, implicitly, the resulting instabilities require a kinetic description in which the details of the velocity distribution are important.

Linear and quasilinear regimes of the electromagnetic instabilities have been extensively investigated, many of the pioneering works presenting theories and simulation experiments of the cyclotron instabilities driven by anisotropic plasma particles (e.g., electrons or protons) with 
standard bi-Maxwellian distributions (Sagdeev and Shafranov, 1961; Vedenov, Velikhov, and Sagdeev, 1961; Trakhtengerts, 1963; Kennel and Petschek, 1966; Davidson et al., 1972; Davidson and Hammer, 1972; Gary and Feldman, 1978). More recent studies (Yoon and Seough, 2012; Seough and Yoon, 2012; Seough et al., 2013) have refined the quasilinear methods to construct the instability threshold conditions as inverse correlations between the temperature anisotropy $A=T_{\perp} / T_{\|}$and parallel plasma beta $\beta_{\|}$, which are usually provided by a linear approach. Moreover, the empirical quasilinear models have been found to provide a useful short-cut research method for a selfconsistent description of the temporal development of cyclotron instabilities (Yoon et al., 2012).

In the present paper we elaborate a quasilinear approach for the electromagnetic cyclotron instabilities in bi-Kappa distributed plasmas. Kappa distribution functions are generalized power-laws which have been successfully employed to reproduce the velocity distributions of plasma particles in the solar wind, see the reviews by Pierrard and Lazar (2010) and Lazar, Schlickeiser, and Poedts (2012). On the other hand, the electromagnetic cyclotron fluctuations are an important component of the space plasma dynamics and their saturation amplitude is particularly important for understanding their implications (e.g., wave-particle interactions leading to particle energization or particle relaxation). Linear dispersion studies of the cyclotron instabilities in bi-Kappa plasmas have revealed certain effects of the suprathermal populations (quantified by finite values of the power-index $\kappa$ ) on the instability thresholds, wave-frequency and growth rates (Summers and Thorne, 1991; Xue, Thorne, and Summers, 1993, 1996; Lazar, Poedts, and Schlickeiser, 2011; Lazar, 2012; Lazar, Poedts, and Michno, 2013; Lazar and Poedts, 2014). These results must be confirmed by a quasilinear approach, and will be reiterated in our analysis in the next section. For the electromagnetic electron cyclotron (EMEC) instability confirmations have already been obtained from numerical simulations with particle-in-cell (PIC) (Lu, Zhou, and Wang, 2010) and Vlasov solvers (Eliasson and Lazar, 2015). The instability saturates (weakly-)nonlinearly by reshaping the velocity distributions and reducing the temperature anisotropy until marginal stablity is reached. PIC simulations have also been used to model the electromagnetic ion (proton) EMIC instability but only for bi-Maxwellian plasmas (Seough, Yoon, and Hwang, 2014).

Here we treat in a similar manner both the EMEC and EMIC instabilities that may develop in bi-Kappa distributed plasmas. The quasilinear theory of these two instabilities is built in section II based on the insights on temporal evolution of the electron velocity distribution gained from the Vlasov simulations of the EMEC instability. We first investigate the quasilinear development of EMEC instability and discuss the temporal evolution of the dynamical electron quantities, i.e., plasma betas $\beta_{\perp}$, $\beta_{\|}$(where $\beta=8 \pi n k_{B} T / B_{0}^{2}$ defines the ratio between the mean kinetic energy density and the mean magnetic field energy density), the temperature anisotropy $A_{e} \equiv T_{e \perp} / T_{e \|}=\beta_{e \perp} / \beta_{e \|}$, and the EMEC wave magnetic field energy density. Then we present a quasilinear approach for the EMIC instability including a temporal analysis of the ion (proton) plasma betas $\beta_{i \perp}, \beta_{i \|}$, the temperature anisotropy $A_{i} \equiv T_{i \perp} / T_{i \|}=\beta_{i \perp} / \beta_{i \|}$, and the EMIC wave magnetic field energy density. Finally, our results are summarized and discussed in section III.

\section{ELECTROMAGNETIC CYCLOTRON INSTABILITIES}

Linear theory predicts a destabilization of the electromagnetic cyclotron modes in magnetized plasmas with anisotropic temperatures $T_{\perp}>T_{\|}$. Kennel and Petschek (1966) have shown that for anisotropic velocity distributions decreasing monotonically with increasing the particle speed the cyclotron modes propagating parallel to the magnetic field $\left(\mathbf{k} \| \mathbf{B}_{0}\right)$ are the most unstable. Since this is a characteristic of any of the bi-Maxwellian or bi-Kappa distribution functions we choose to investigate only the parallel $\left(k_{\|}=k\right)$ cyclotron instabilities.

\section{A. Insights from Vlasov simulations}

Information provided by linear response theory may be of particular importance for numerical experiments. Thus, the fastest growing modes often invoked in simulations correspond to the instability maximum growthrates derived within a linear approach. In turn, a quasilinear (or nonlinear) theory is usually conditioned by insights gained from numerical simulations, which indicate realistic models for the evolution of plasma parameters.

Here we extend the numerical kinetic (Vlasov) analysis of the EMEC instability from Eliasson and Lazar (2015) by looking into details of the evolution of electron velocity distribution. The initial anisotropic distributions of electrons are modelled by a bi-Kappa distribution in the same geometry assuming the background magnetic field $\mathbf{B}_{0}=\hat{\mathbf{z}} B_{0}$ directed along the $z$-axis. Linear dispersion and stability formalism as well as the numerical setup for a temporal resolution are described in the paper work by Eliasson and Lazar (2015) and will not be repeated here. Figures 1-4 present the results from four Vlasov runs with different plasma parameters. Left column display temporal profiles of the parallel distribution function (distribution function integrated over perpendicular velocity space) assuming initially a bi-Kappa either with $\kappa=2$ (Figures 1 and 2) or with $\kappa=4$ (Figures 3 and 4 ). We have also combined two distinct sets of plasma plasma parameters: $\beta_{e \|}=0.128, T_{e \perp} / T_{e \|}=8$, $k_{B} T_{e \|}=m_{e} c^{2} / 2500, \omega_{p e} / \Omega=12.65$ in Figures 1 and 3 and $\beta_{e \|}=1.28, T_{e \perp} / T_{e \|}=4, k_{B} T_{e \|}=m_{e} c^{2} / 2500$, $\omega_{p e} / \Omega=40$ in Figures 2 and 4 , where $\Omega=\left|\Omega_{e}\right|$. Each figure displays nine different instants in time of the parallel distribution from the initiation and until the satu- 

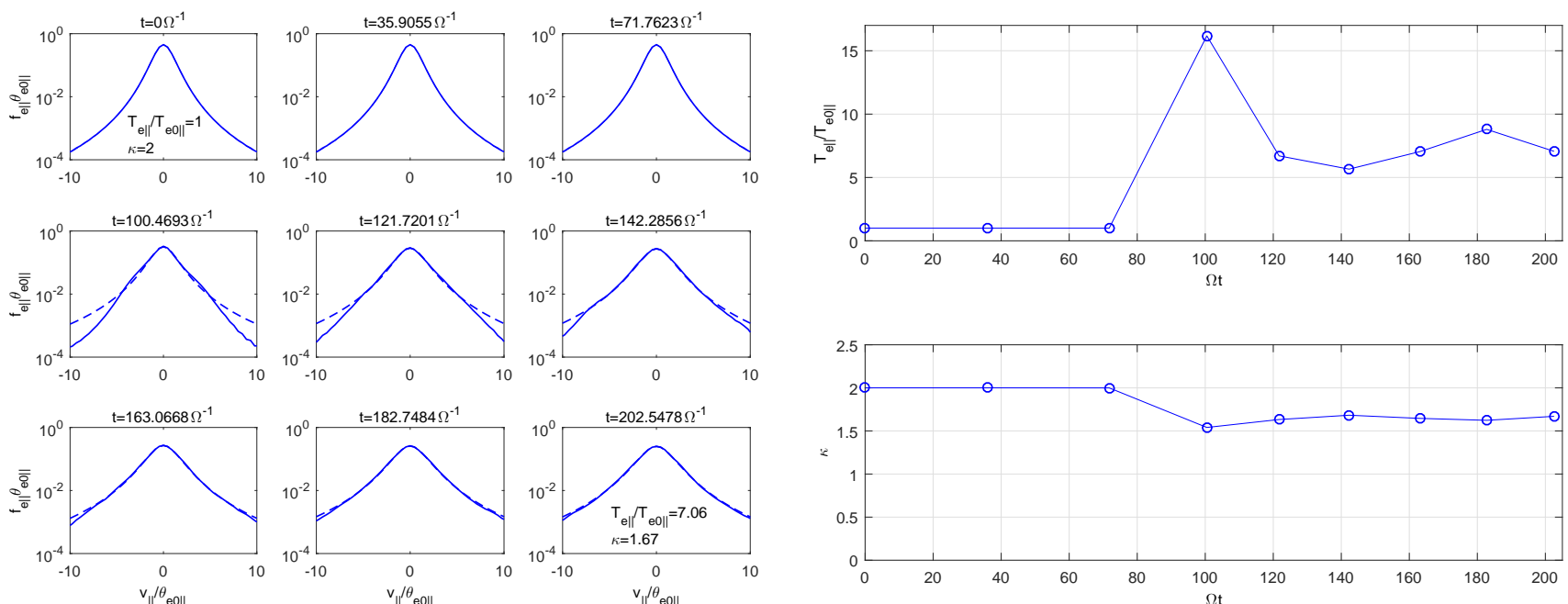

FIG. 1. (Left) Profiles of the distribution function (in parallel direction) at nine different instants in time (indicated above each panel) from a Vlasov simulation setup: $\kappa=2, \beta_{e \|}=0.128, T_{e \perp} / T_{e \|}=8, k_{B} T_{e \|}=m_{e} c^{2} / 2500, \omega_{p e} / \Omega=12.65$. The dashed lines are the numerical fits. (Right) Temporal evolution for the parallel temperature (top panel) and power-index $\kappa$ (bottom panel) for the same run.
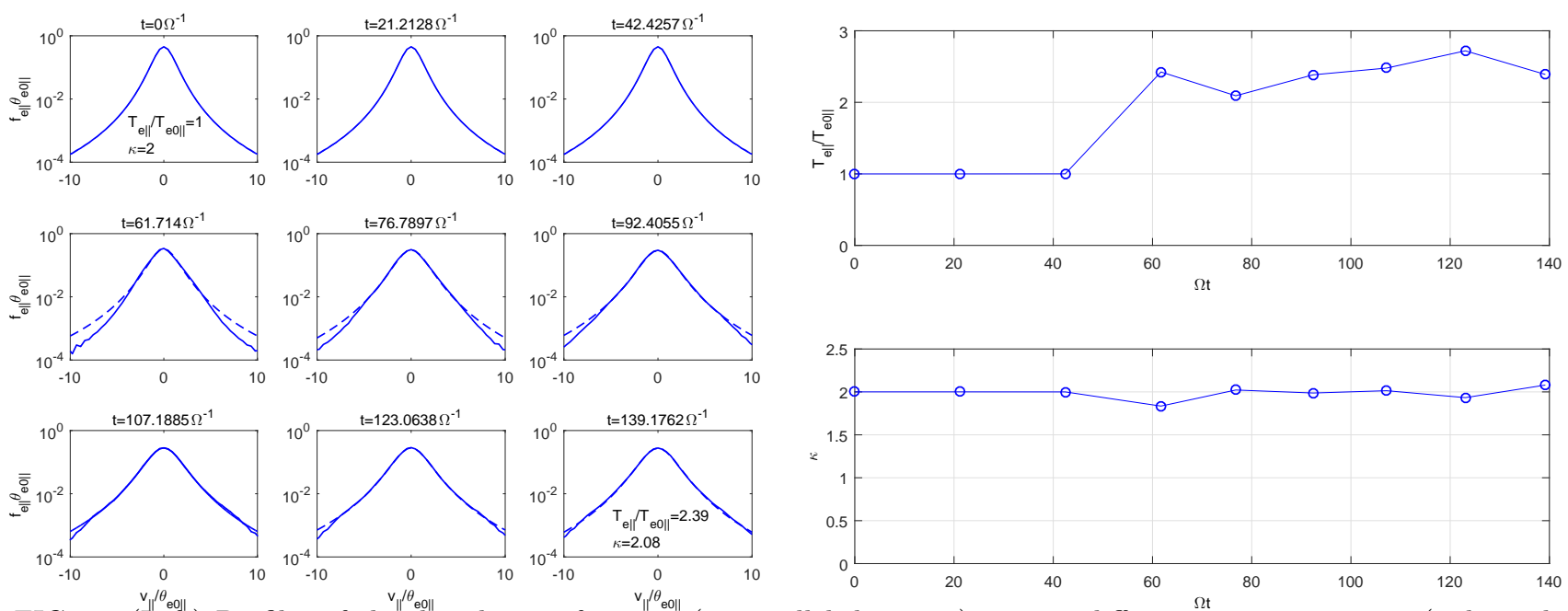

FIG. 2. (Left) Profiles of the distribution function (in parallel direction) at nine different instants in time (indicated above each panel) from a Vlasov simulation setup: $\kappa=2, \beta_{e \|}=1.28, T_{e \perp} / T_{e \|}=4, k_{B} T_{e \|}=m_{e} c^{2} / 2500, \omega_{p e} / \Omega=40$. The dashed lines are the numerical fits. (Right) Temporal evolution for the parallel temperature (top panel) and power-index $\kappa$ (bottom panel) for the same run.

ration of the instability. Numerical fits are made using a one-dimensional Kappa distribution function (dashed lines in the left panels of Figs. 1-4) to obtain estimates of the parallel temperature and power-index, shown in the right panels of Figs. 1-4. An increase of the parallel temperature becomes obvious in both cases.

Quantitatively, this is confirmed by the temporal evolution of the parallel electron temperature (normalized by the initial temperature) in the right column of each figure (top panels). Bottom panels in the right column present temporal evolution of the power-index $\kappa$. Interesting for our present purpose is the relative constancy shown by $\kappa$, with an exception in the second run, when it shows a transient spike at $t=42.0678 \Omega^{-1}$, which is due to that the low-energy electrons are heated first (in the parallel direction) making the distribution function more similar to a Maxwellian with an increased $\kappa$, and only later are the high-energy electrons also heated so that $\kappa$ decreases to its initial value. Similar as for the bi-Maxwellian case (Davidson et al., 1972), it was observed in the Vlasov simulations that the nonlinear stabilization of modes occurred sequentially from higher to lower wavenumbers so that the wave spectrum shifted towards longer wavelengths at later times. This might explain the 

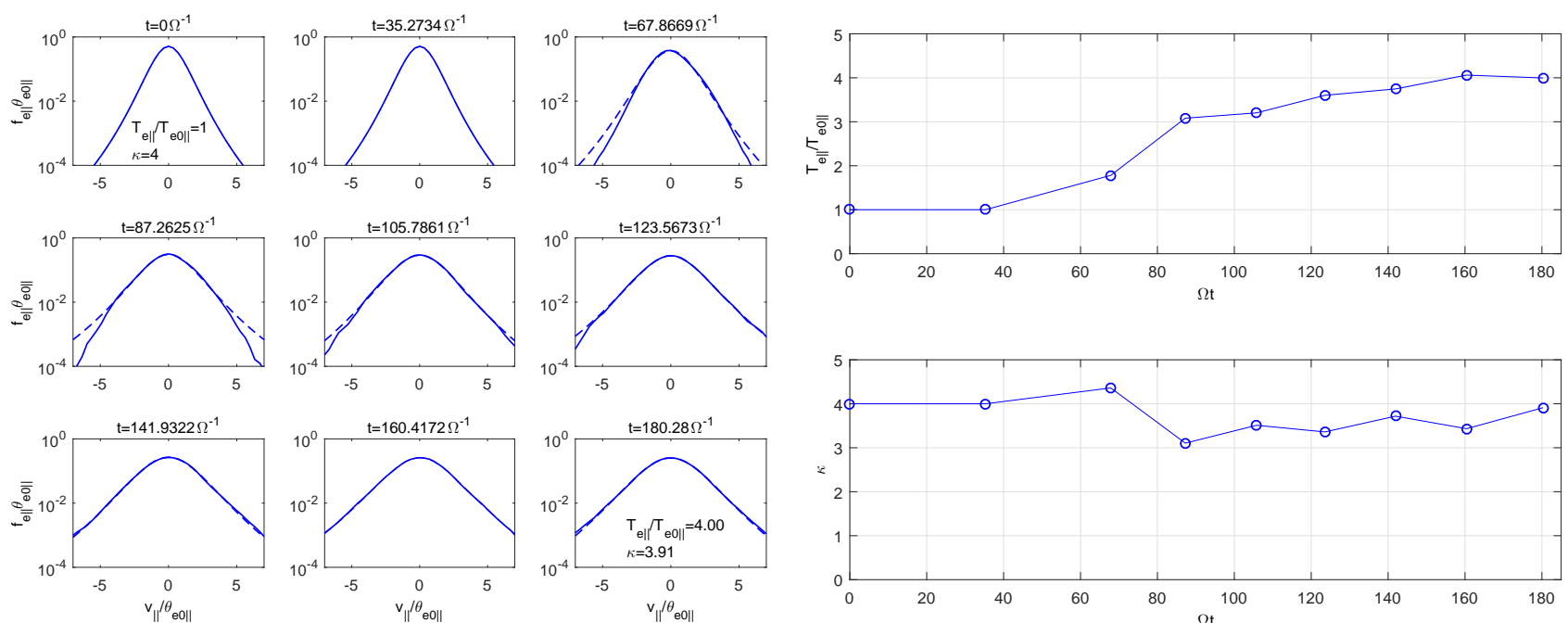

FIG. 3. (Left) Profiles of the distribution function (in parallel direction) at nine different instants in time (indicated above each panel) from a Vlasov simulation setup: $\kappa=4, \beta_{e \|}=0.128, T_{e \perp} / T_{e \|}=8, k_{B} T_{e \|}=m_{e} c^{2} / 2500, \omega_{p e} / \Omega=12.65$. The dashed lines are the numerical fits. (Right) Temporal evolution for the parallel temperature (top panel) and power-index $\kappa$ (bottom panel) for the same run.
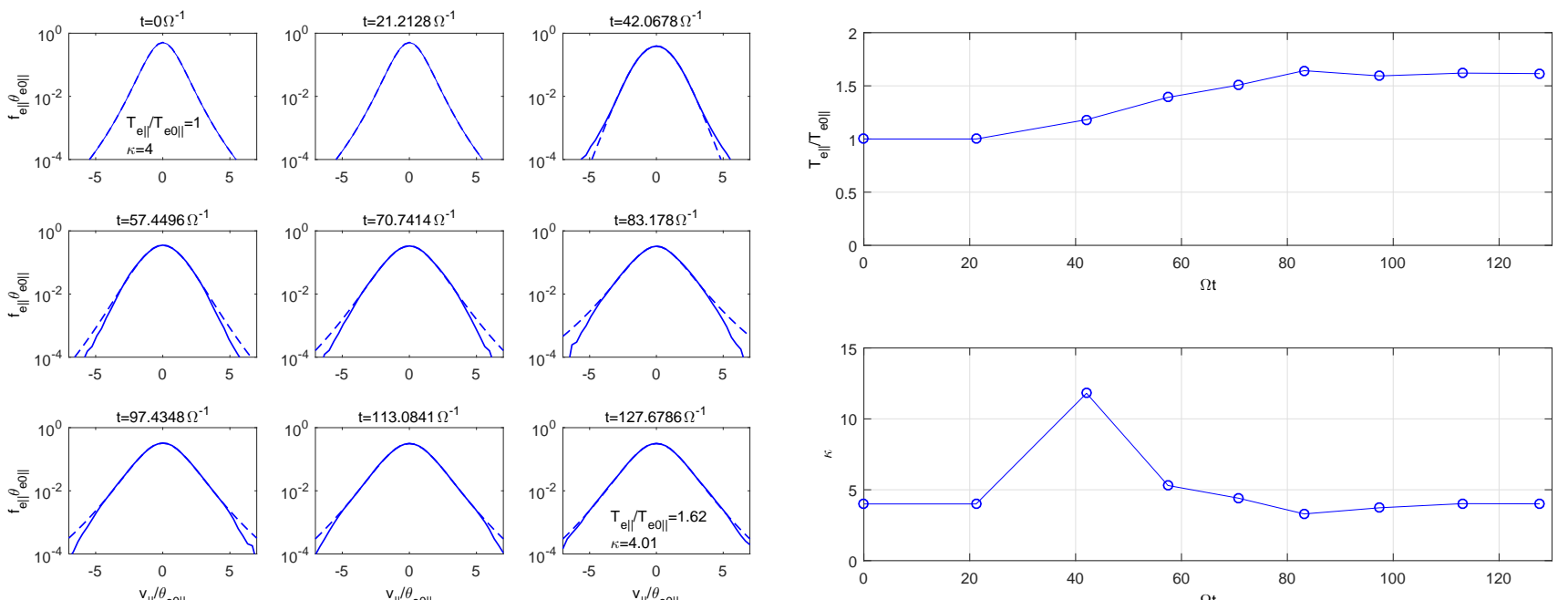

FIG. 4. (Left) Profiles of the distribution function (in parallel direction) at nine different instants in time (indicated above each panel) from a Vlasov simulation setup: $\kappa=4, \beta_{e \|}=1.28, T_{e \perp} / T_{e \|}=4, k_{B} T_{e \|}=m_{e} c^{2} / 2500, \omega_{p e} / \Omega=40$. The dashed lines are the numerical fits. (Right) Temporal evolution for the parallel temperature (top panel) and power-index $\kappa$ (bottom panel) for the same run.

time-dependent differential heating of the electrons. Hence, the power-index $\kappa$ can be considered almost constant over the time period of quasilinear saturation, and in the next we will assume that the anisotropic bi-Kappa distribution retains the self-similar shape, i.e., it holds the power-index $\kappa$ constant, but the macroscopic properties like temperature components, and, implicitly, the temperature anisotropy are allowed to change under the influence of growing fluctuations.

\section{B. Quasilinear approach}

The wave frequency $(\omega)$ of the cyclotron modes is sufficiently low, $\omega^{2} \ll k^{2} c^{2}$, such that we can neglect the displacement current in Maxwell's equations, and from linear theory we find the general form of the dispersion relation for the parallel electromagnetic waves

$$
\begin{aligned}
1= & \pi \sum_{s} \frac{\omega_{p s}}{k^{2} c^{2}} \int_{-\infty}^{\infty} \frac{d v_{\|}}{\omega-k v_{\|} \pm \Omega_{s}} \\
& \times \int_{0}^{\infty} d v_{\perp} v_{\perp}\left[k v_{\perp}^{2} \frac{\partial f_{s}}{\partial v_{\|}}-2\left(\omega-k v_{\|}\right) f_{s}\right]
\end{aligned}
$$


for an arbitrary distribution function $f_{s}$ of particles of species $s$ ( $a=e$ for electrons and $a=p$ for protons $)$, where $\omega_{p s}=\left(4 \pi n e_{s}^{2} / m_{s}\right)^{1 / 2}$ is the plasma frequency, and " \pm " state for the right-handed (RH) or left-handed (LH) circularly polarized modes. In magnetized plasmas the velocity distributions can be assumed gyrotropic and we can consider polar coordinates $v_{\perp}=\left(v_{x}^{2}+v_{y}^{2}\right)^{1 / 2}$ and $v_{\|}=v_{z}$.

In the second-order (diffusion) approximation the equation for particles reads

$$
\begin{aligned}
\frac{\partial f_{s}}{\partial t}= & \frac{i e_{s}^{2}}{4 m_{s}^{2}} \int_{-\infty}^{\infty} d k \int_{-\infty}^{\infty} d \omega \sum_{+,-} \frac{1}{v_{\perp}}\left[\left(1-\frac{k v_{\|}}{\omega^{*}}\right) \frac{\partial}{\partial v_{\perp}}\right. \\
& \left.+\frac{k v_{\perp}}{\omega^{*}} \frac{\partial}{\partial v_{\|}}\right] v_{\perp} \frac{<\left|\delta E_{x}(k, \omega) \mp \delta E_{y}(k, \omega)\right|^{2}>}{\omega-k v_{\|} \pm \Omega_{s}} \\
& \times\left[\left(1-\frac{k v_{\|}}{\omega}\right) \frac{\partial f_{s}}{\partial v_{\perp}}+\frac{k v_{\perp}}{\omega} \frac{\partial f_{s}}{\partial v_{\|}}\right] .
\end{aligned}
$$

For the second-order moments of the distribution (defining the temperature components), after integration we obtain

$$
\begin{aligned}
\frac{d T_{s \perp}}{d t} \equiv & \frac{m_{s}}{2 k_{B}} \frac{\partial}{\partial t} \int d \mathbf{v} v_{\perp}^{2} f_{s}=\frac{i \pi e_{s}^{2}}{2 m_{s} k_{B}} \int_{-\infty}^{\infty} d k \int_{-\infty}^{\infty} d \omega \\
& \sum_{+,}<\left|\delta E_{x}(k, \omega) \mp \delta E_{y}(k, \omega)\right|^{2}> \\
& \times \int_{-\infty}^{\infty} d v_{\|} \frac{1-k v_{\|} / \omega^{*}}{\omega-k v_{\|} \pm \Omega_{s}} \int_{0}^{\infty} d v_{\perp} v_{\perp} \\
& \times\left[\frac{k v_{\perp}^{2}}{\omega} \frac{\partial f_{s}}{\partial v_{\|}}-2\left(1-\frac{k v_{\|}}{\omega}\right) f_{s}\right] \\
\frac{d T_{s \|}}{d t} \equiv & \frac{m_{s}}{k_{B}} \frac{\partial}{\partial t} \int d \mathbf{v} v_{\|}^{2} f_{s}=\frac{i \pi e_{s}^{2}}{m_{s} k_{B}} \int_{-\infty}^{\infty} d k \int_{-\infty}^{\infty} d \omega \\
& \sum_{+}<\left|\delta E_{x}(k, \omega) \mp \delta E_{y}(k, \omega)\right|^{2}> \\
& \times \int_{-\infty}^{\infty} d v_{\|} \frac{k v_{\|} / \omega^{*}}{\omega-k v_{\|} \pm \Omega_{s}} \int_{0}^{\infty} d v_{\perp} v_{\perp} \\
& \times\left[\frac{k v_{\perp}^{2}}{\omega} \frac{\partial f_{s}}{\partial v_{\|}}-2\left(1-\frac{k v_{\|}}{\omega}\right) f_{s}\right]
\end{aligned}
$$

If $\omega=\omega_{r}+i \gamma$ is the complex root of the dispersion relation, we may define the spectral magnetic field wave energy density

$$
\delta B_{ \pm}^{2}(k)=\frac{k^{2} c^{2}}{|\omega|^{2}}<\left|\delta E_{x}(k, \omega) \mp \delta E_{y}(k, \omega)\right|^{2}>.
$$

\section{EMEC instability}

Before any particularization of the quasilinear equations obtained above, we briefly introduce the bi-Kappa
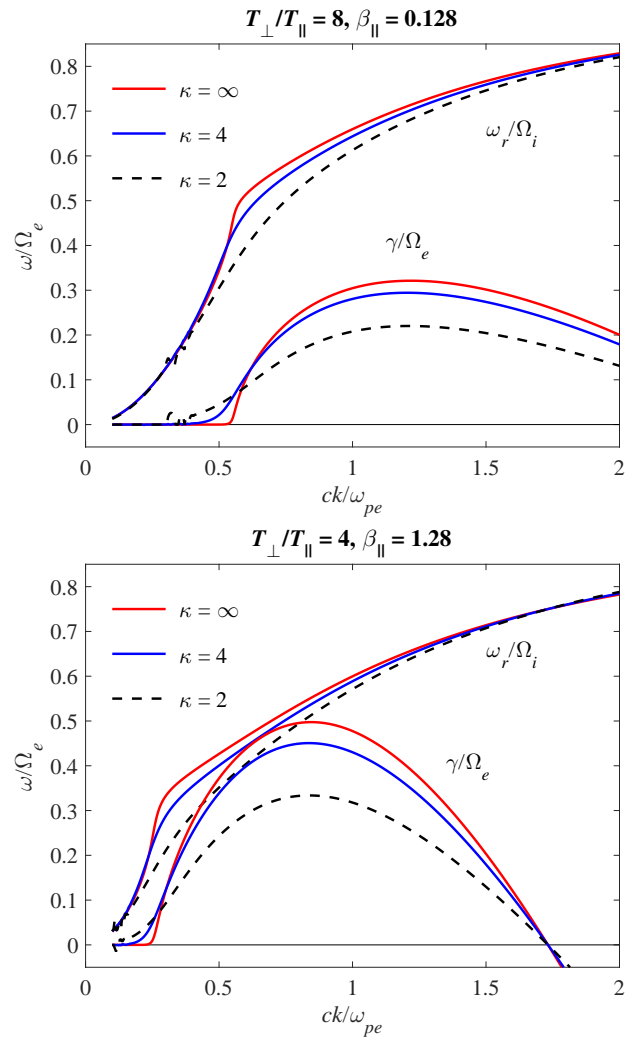

FIG. 5. Wavenumber dispersion of the EMEC instabilities for the two sets of plasma parameters analyzed in Figs. 1-4 (also explicitly given here in each panel): $\omega_{r}=\Re(\omega)$ is the wave-frequency and $\gamma=\Im(\omega)$ is the growth rate.

distribution function

$$
f_{\kappa}=\frac{1}{\pi^{3 / 2} \theta_{\perp}^{2} \theta_{\|}} \frac{\Gamma(\kappa)}{\kappa^{1 / 2} \Gamma(\kappa-1 / 2)}\left(1+\frac{v_{\|}^{2}}{\kappa \theta_{\|}^{2}}+\frac{v_{\perp}^{2}}{\kappa \theta_{\perp}^{2}}\right)^{-\kappa-1}
$$

where the power index must satisfy $\kappa>3 / 2$ to ensure the existence of the parallel and perpendicular thermal speeds $\theta_{\|}$and $\theta_{\perp}$, which are defined in terms of the respective components $T_{\|}$and $T_{\perp}$ of the kinetic temperature

$$
\begin{gathered}
T_{\|}=\frac{m}{k_{B}} \int d \mathbf{v} v_{\|}^{2} f=\frac{m_{e} \theta_{\|}^{2}}{2 k_{B}} \frac{\kappa}{\kappa-3 / 2} \\
T_{\perp}=\frac{m}{2 k_{B}} \int d \mathbf{v} v_{\perp}^{2} f=\frac{m_{e} \theta_{\perp}^{2}}{2 k_{B}} \frac{\kappa}{\kappa-3 / 2} .
\end{gathered}
$$

In the limit $\kappa \rightarrow \infty$, the bi-Kappa distribution converges to a standard bi-Maxwellian distribution function.

The EMEC instability has RH polarization and sufficiently high frequency $\Omega>\omega_{r} \gg \Omega_{p}$, so that we may ignore the proton (ion) response, and the dispersion relation (1) retains only the contribution of electrons assumed to be bi-Kappa distributed (Eliasson and Lazar, 

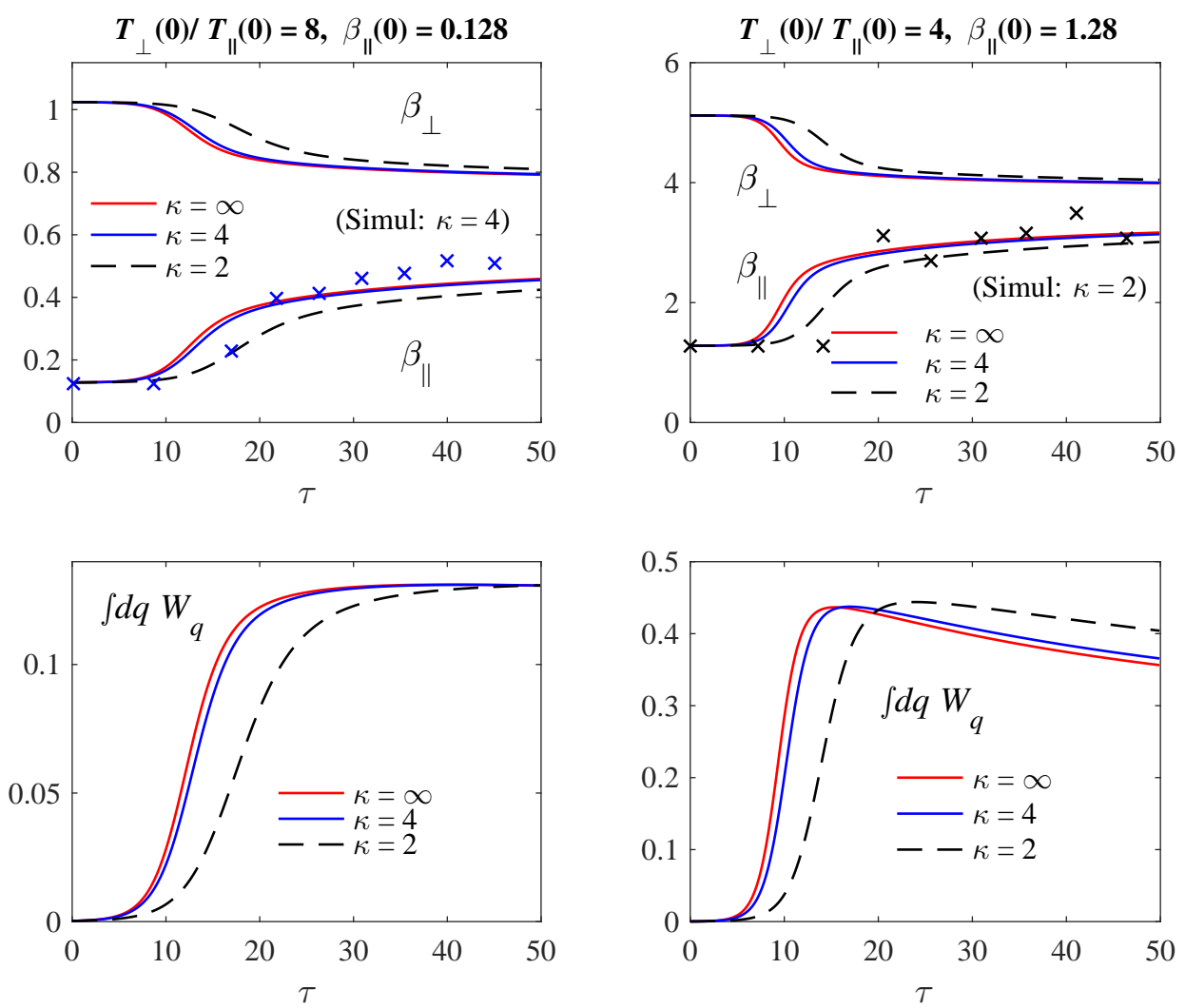

FIG. 6. Time evolutions from quasilinear approach for $\beta_{e \perp}$ and $\beta_{e \|}$ (top) and for the EMEC fluctuating magnetic field energy (bottom). Initial plasma conditions are those invoked in Fig. 5. The results from two Vlasov runs are shown with crosses in top panels.

2015)

$\frac{k^{2} c^{2}}{\omega_{p e}^{2}}+1=\frac{T_{e \perp}}{T_{e \|}}+\left(\frac{T_{e \perp}}{T_{e \|}} \frac{\omega-\Omega}{k \theta_{e \|}}+\frac{\Omega}{k \theta_{e \|}}\right) Z_{\kappa}\left(\frac{\omega-\Omega}{k \theta_{e \|}}\right)$,

which is written in terms of the dispersion function modified for Kappa distributed plasmas (Lazar, Schlickeiser, and Shukla, 2008)

$$
\begin{array}{r}
Z_{\kappa}(\xi)=\frac{1}{\pi^{1 / 2} \kappa^{1 / 2}} \frac{\Gamma(\kappa)}{\Gamma(\kappa-1 / 2)} \int_{-\infty}^{\infty} d x \frac{\left(1+x^{2} / \kappa\right)^{-\kappa}}{x-\xi} \\
\Im(\xi)>0 .
\end{array}
$$

Figure 5 presents the unstable EMEC solutions obtained for the two sets of plasma parameters discussed above in Figs. 1 and 2. The wave-frequencies $\left(\omega_{r}=\Re(\omega)\right)$ and growth-rates $(\gamma=\Im(\omega))$ are derived for finite values of the power-index, e.g., $\kappa=2,4$ and for the limit (Maxwellian) case $\kappa \rightarrow \infty$. If the temperature is maintained independent of $\kappa$, i.e, $T_{e}=T_{e, \kappa}=T_{e, \kappa \rightarrow \infty}$ (where $T_{e, \kappa \rightarrow \infty}$ is associated to the Maxwellian limit), the growth-rates are found to be diminished (instability is inhibited) in the presence of suprathermal populations, i.e., with decreasing the values of $\kappa$. These effects become significant with decreasing $\kappa$ to the low values approaching the limit $\kappa>3 / 2$, and are in perfect agreement with the earlier results on EMEC instability in bi-Kappa plasmas (Lazar, Poedts, and Michno, 2013; Lazar, Poedts, and Schlickeiser, 2011; Eliasson and Lazar, 2015).

From Eqs. (3)-(5), keeping only the real part, the (macroscopic) quasilinear equations for the electron temperature components become

$$
\frac{d T_{e \perp}}{d t}=-\frac{e^{2}}{m_{e} k_{B}} \int_{-\infty}^{\infty} \frac{d k}{c^{2} k^{2}} \gamma\left(\frac{c^{2} k^{2}}{\omega_{p e}^{2}}+\frac{1}{2}\right) \delta B^{2}(k),
$$

$$
\frac{d T_{e \|}}{d t}=\frac{e^{2}}{m_{e} k_{B}} \int_{-\infty}^{\infty} \frac{d k}{c^{2} k^{2}} \gamma\left(\frac{c^{2} k^{2}}{\omega_{p e}^{2}}+1\right) \delta B^{2}(k) .
$$

where $\gamma=\gamma(k)$ is solution of the dispersion relation (9), and the growing fluctuations are described by the wave kinetic equation

$$
\frac{\partial}{\partial t} \delta B^{2}(k)=2 \gamma \delta B^{2}(k)
$$

Experience indicates that solutions are simpler in terms of dimensionless quantities $\beta_{e \|, \perp}=8 \pi n k_{B} T_{e \|, \perp} / B_{0}^{2}$, 
$A_{e}=T_{e \perp} / T_{e \|}=\beta_{e \perp} / \beta_{e \|}, q=c k / \omega_{p e}, w_{q}=\omega(k) / \Omega$, $\gamma_{q}=\gamma(k) / \Omega, \tau=t \Omega$ and $W_{q}=\delta B^{2}(k) / B_{0}^{2}$, for which the quasilinear equations become

$$
\begin{gathered}
\frac{d \beta_{e \perp}}{d \tau}=-2 \int_{-\infty}^{\infty} d q\left(1+\frac{1}{2 q^{2}}\right) \gamma_{q} W_{q}, \\
\frac{d \beta_{e \|}}{d \tau}=2 \int_{-\infty}^{\infty} d q\left(1+\frac{1}{q^{2}}\right) \gamma_{q} W_{q},
\end{gathered}
$$

and

$$
\frac{\partial}{\partial \tau} W_{q}=2 \gamma_{q} W_{q}
$$

This set of equations can be closed by adding the dispersion relation (9)

$$
\begin{aligned}
q^{2}+1= & A_{e}+\frac{A_{e}\left(w_{q}+i \gamma_{q}\right)-A_{e}+1}{q \beta_{\|}^{1 / 2}}\left(\frac{\kappa}{\kappa-3 / 2}\right)^{1 / 2} \\
& \times Z_{\kappa}\left[\frac{w_{q}+i \gamma_{q}-1}{q \beta_{e \|}^{1 / 2}}\left(\frac{\kappa}{\kappa-3 / 2}\right)^{1 / 2}\right]
\end{aligned}
$$

Eqs. (14)-(17) represent a system of coupled nonlinear equations that must be solved simultaneously to find all the unknown variables. We do this numerically, and for the EMEC unstable solutions linearly described in Fig. 5 the quasilinear time evolution of the macroscopic quantities $\beta_{e \perp}$ and $\beta_{e \|}$, and the saturation of the magnetic wave energy density $\left(W_{q}\right)$ are shown in Fig. 6 . The time of relaxation of the anisotropy corresponds to the time of saturation of the instability. For $\kappa=2$ this time is more than 10 (dimensionless) units longer that that obtained in the Maxwellian limit $\kappa \rightarrow \infty$. For comparison, with crosses (top panels) we show the results from two Vlasov runs in Figs. 2 and 4, where the power-index $\kappa$ remains almost constant. The assumption of a constant $\kappa$ seems to break down in the combination of small $\kappa$ and large temperature anisotropies in the other two cases in Figs. 1 and 3.

\section{EMIC instability}

The EMIC modes have LH polarization and even lower frequencies $\omega_{r}<\Omega_{i}$. The protons are assumed biKappa distributed, see Eqs. (6)-(8), while the electrons are treated as isotropic and cold, so that their contribution in a charge neutral plasma can be reduced to $\omega_{p e}^{2} / \Omega_{e}=\omega_{p i}^{2} / \Omega_{i}$, and then dispersion relation (1) simplifies to

$$
\begin{aligned}
\frac{c^{2} k_{\|}^{2}}{\omega_{p i}^{2}}+\frac{\omega}{\Omega_{i}}+1=\frac{T_{\perp}}{T_{\|}} & +\left[\frac{T_{\perp}}{T_{\|}} \frac{\omega-\Omega_{i}}{k_{\|} \theta_{\|}}+\frac{\Omega_{i}}{k_{\|} \theta_{\|}}\right] \\
& \times Z_{\kappa}\left(\frac{\omega-\Omega_{i}}{k_{\|} \theta_{\|}}\right)
\end{aligned}
$$

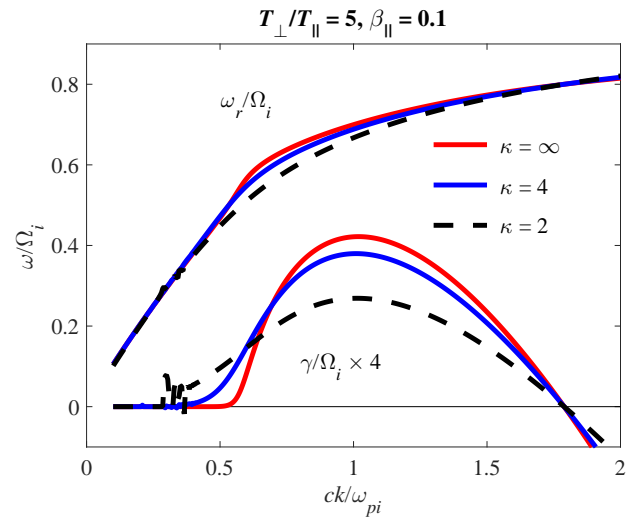

FIG. 7. Wavenumber dispersion of the EMIC instabilities for finite values of the power-index $\kappa=2,4$ and for the Maxwellian limit $\kappa \rightarrow \infty: \omega_{r}=\Re(\omega)$ is the wave-frequency and $\gamma=\Im(\omega)$ is the growth rate. Proton parameters are indicated on top of the picture frame.

in terms of the same Kappa dispersion function from Eq. (10). We omit the subscript " $i$ " for the temperature and thermal velocity of the ions (protons) as only this species is anisotropic, with $T_{\perp}>T_{\|}$. Figure 7 presents the unstable EMIC solutions obtained for a set of proton parameters $T_{\perp} / T_{\|}=5$ and $\beta_{\|}=0.1$. For comparison, the wave-frequencies $\left(\omega_{r}=\Re(\omega)\right)$ and growth-rates $(\gamma=\Im(\omega))$ are derived for two finite values of the powerindex, e.g., $\kappa=2,4$ and for the Maxwellian limit $\kappa \rightarrow \infty$. If the temperature is maintained independent of $\kappa$, i.e, $T=T_{\kappa}=T_{\kappa \rightarrow \infty}$ the EMIC growth-rates are diminished (instability is inhibited) in the presence of suprathermal populations, i.e., with decreasing the values of $\kappa$. This effect is similar to the one obtained for the EMEC instabilities and is in total agreement with the previous studies of the EMIC instability in bi-Kappa distributed plasmas (Lazar, 2012; Lazar and Poedts, 2014).

For the quasilinear evolution of the proton distribution we assume that the power-index kappa does not change signficantly, as was shown in Vlasov simulations for the EMEC instability. In order to describe the velocity distribution and the fluctuating magnetic field we use again the general equations (3)-(5), and for the proton temperature components (keeping only the real part) we obtain

$$
\frac{d T_{\perp}}{d t}=-\frac{e^{2}}{m_{i} k_{B}} \int_{-\infty}^{\infty} \frac{d k}{c^{2} k^{2}} \gamma\left(\frac{c^{2} k^{2}}{\omega_{p i}^{2}}+\frac{\omega_{r}}{\Omega_{i}}\right) \delta B^{2}(k),
$$

$$
\frac{d T_{\|}}{d t}=\frac{e^{2}}{m_{i} k_{B}} \int_{-\infty}^{\infty} \frac{d k}{c^{2} k^{2}} \gamma\left(\frac{c^{2} k^{2}}{\omega_{p i}^{2}}+\frac{2 \omega_{r}}{\Omega_{i}}\right) \delta B^{2}(k) .
$$

where $\omega_{r}=\omega_{r}(k)$ and $\gamma=\gamma(k)$ are the real and imaginary solutions of the dispersion relation (18). Growing fluctuations are described by the wave kinetic equation

$$
\frac{\partial}{\partial t} \delta B^{2}(k)=2 \gamma \delta B^{2}(k)
$$



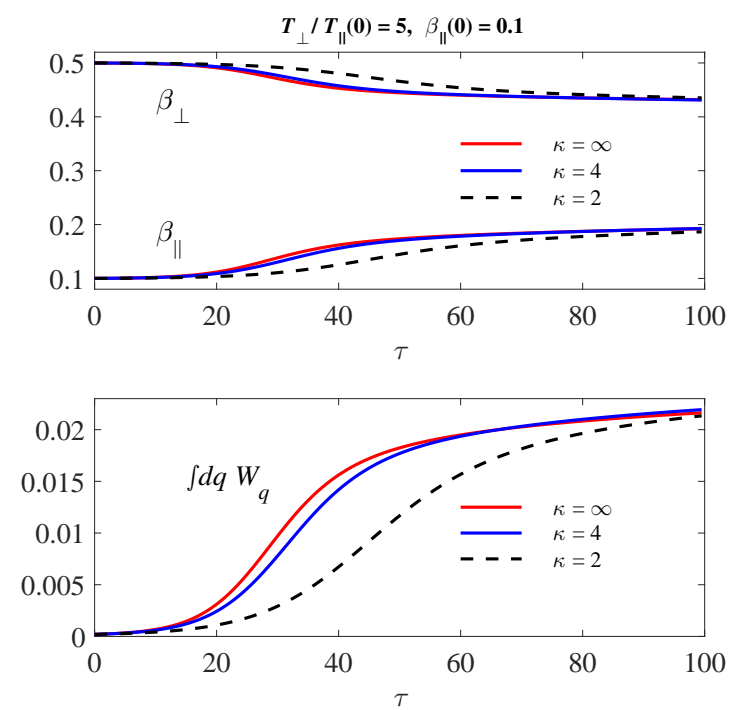

FIG. 8. Time evolutions for $\beta_{\perp}$ and $\beta_{\|}$(top) and for the EMIC fluctuating magnetic field energy (bottom). Initial plasma conditions are those invoked in Fig. 7.

We invoke again the dimensionless quantities $\beta_{\|, \perp}=$ $8 \pi n k_{B} T_{\|, \perp} / B_{0}^{2}, A=T_{\perp} / T_{\|}=\beta_{\perp} / \beta_{\|}, q=c k / \omega_{p i}, w_{q}=$ $\omega(k) / \Omega, \gamma_{q}=\gamma(k) / \Omega_{i}, \tau=t \Omega_{i}$ and $W_{q}=\delta B^{2}(k) / B_{0}^{2}$, and find for the set of quasilinear equations

$$
\begin{gathered}
\frac{d \beta_{\perp}}{d \tau}=-2 \int_{-\infty}^{\infty} d q\left(1+\frac{w_{q}^{2}}{2 q^{2}}\right) \gamma_{q} W_{q}, \\
\frac{d \beta_{\|}}{d \tau}=2 \int_{-\infty}^{\infty} d q\left(1+\frac{2 w_{q}^{2}}{q^{2}}\right) \gamma_{q} W_{q} \\
\frac{\partial}{\partial \tau} W_{q}=2 \gamma_{q} W_{q}
\end{gathered}
$$

completed by the dispersion relation (18)

$$
\begin{aligned}
0=q^{2}+w_{q}+i \gamma_{q}-A-\frac{(A+1)\left(w_{q}+i \gamma_{q}\right)-A}{q \beta_{\|}^{1 / 2}} \\
\quad \times\left(\frac{\kappa}{\kappa-3 / 2}\right)^{1 / 2} Z_{\kappa}\left[\frac{w_{q}+i \gamma_{q}-1}{q \beta_{\|}^{1 / 2}}\left(\frac{\kappa}{\kappa-3 / 2}\right)^{1 / 2}\right] .
\end{aligned}
$$

We deal with another system of coupled nonlinear equations (22)-(25) which can be solved only numerically. For the EMIC unstable solutions linearly described in Fig. 7 the quasilinear time evolution of the macroscopic quantities $\beta_{\perp}$ and $\beta_{\|}$and the saturation of the magnetic wave energy density $\left(W_{q}\right)$ are shown in Fig. 8 . The time of saturation is markedly increased with decreasing $\kappa$, the shortest saturation time being obtained in the Maxwellian limit.

\section{SUMMARY}

In this paper we have presented a quasilinear approach for the electromagnetic cyclotron instabilities in bi-Kappa distributed plasmas. Natural space plasmas display non-Maxwellian high-energy tail distributions which are described by the generalized Kappa powerlaws. We have studied both the electromagnetic electron cyclotron (EMEC) instability and the electromagnetic ion (proton) cyclotron (EMIC) unstable modes. A quasilinear investigation enables to understand and quantify the efficiency of free energy transfer from anisotropic particles to their electromagnetic perturbations under the development of the instability, and, vice-versa, the energy transferred from the resulting wave fluctuations back to particles under the saturation of the instability. Responsible for this transfer in both directions is the resonant interaction of plasma particles with the electromagnetic cyclotron modes.

The quasilinear approach is built on premises indicated by the Vlasov simulations, which show that the value of the power-index $\kappa$ does not change significantly during the development and saturation of the EMEC instability. Relevant in this sense are the four sets of results displayed in Figs. 1-4, where the right-bottom panels show the time variation of $\kappa$-index. From the quasilinear development of the EMEC instability, the time evolutions of the electron kinetic energy densities normalized to the magnetic field energy density, e.g., $\beta_{\|}$and $\beta_{\perp}$, are shown in Figs. 6 (top panels) for the same sets of plasma parameters invoked in Vlasov simulations. If the number of particles in the system is conserved, the temperature components $T_{\|}$ and $T_{\perp}$ follow the same variations, namely, an increase of the parallel component accompanied by a decrease of the parallel temperature. The decrease of the temperature anisotropy is responsible for the saturation of the wave growth, which is shown in the same figure, bottom panels, in terms of the wave magnetic field energy density. For the anisotropic protons with $T_{\perp}>T_{\|}$a very similar behavior is obtained under the back reaction of the EMIC instability, and one relevant example is shown in Fig. 8. Assuming the temperatures of the anisotropic particles independent of $\kappa$, both the EMEC and EMIC instabilities are found to be inhibited by the suprathermal populations, and implicitly the saturation processes slow down with increasing the presence of suprathermals, i.e., with decreasing power-index $\kappa$.

The Vlasov simulations showing the evolution of the particle distributions and their main properties may provide valuable validations for the results from quasilinear theory. Fig. 6 provides a direct comparison with two Vlasov runs in Figs. 2 and 4, where the power-index $\kappa$ remains almost constant. In the other two runs presented in Figs. 1 and 3 the assumption of a constant $\kappa$ seems to break down due to the combination of small $\kappa$ and large temperature anisotropies. Lu, Zhou, and Wang (2010) have also found a decrease of $\kappa$ - 
index in PIC simulations of the same instability driven by large temperature anisotropies. More elaborate comparative analyses involving the results from Vlasov and PIC simulations will be subject of future investigations. The quasilinear formalism presented here is particularly important, as it can offer an intuitive guiding model for studying other kinetic instabilities, like firehose and mirror instabilities, which can develop in bi-Kappa distributed plasmas from space.

\section{ACKNOWLEDGMENTS}

M.L. acknowledges support from the Katholieke Universiteit Leuven, Ruhr-University Bochum and Alexander von Humboldt Foundation. These results were obtained in the framework of the projects G0A2316N (FWO-Vlaanderen) and SCHL 201/35-1 (DFG-German Research Foundation). P.H.Y. acknowledges NSF grant AGS1550566 to the University of Maryland, and the BK21 plus program from the National Research Foundation (NRF), Korea, to Kyung Hee University. He also acknowledges the Science Award Grant from the GFT, Inc., to the University of Maryland. B.E. acknowledges support from the Engineering and Physical Sciences Research Council (EPSRC), UK, Grant No. EP/M009386/1. The Vlasov simulation data associated with this paper are available at: http://dx.doi.org//10.15129/cd90b629-a2b34c75-8c22-58abe72ad03c.

Alexandrova, O., "Solar wind vs magnetosheath turbulence and Alfvén vortices," Nonlinear Processes in Geophysics 15, 95-108 (2008).

Bauer, T. M., Baumjohann, W., Treumann, R. A., Sckopke, N., and Lühr, H., "Low-frequency waves in the near-Earth plasma sheet," JGR 100, 9605-9618 (1995).

Borovsky, J. E. and Funsten, H. O., "MHD turbulence in the Earth's plasma sheet: Dynamics, dissipation, and driving," Journal of Geophysical Research (Space Physics) 108, 1284 (2003).

Brice, N., "An explanation of triggered very-low-frequency emissions," JGR 68, 4626 (1963).

Bruno, R. and Carbone, V., "The solar wind as a turbulence laboratory," Living Reviews in Solar Physics 10 (2013), 10.1007/lrsp2013-2.

Cargill, P. J., Lavraud, B., Owen, C. J., Grison, B., Dunlop, M. W., Cornilleau-Wehrlin, N., Escoubet, C. P., Paschmann, G., Phan, T. D., Rezeau, L., Bogdanova, Y., and Nykyri, K., "Cluster at the Magnetospheric Cusps," SSREV 118, 321-366 (2005).

Davidson, R. C. and Hammer, D. A., "Nonequilibrium Energy Constants Associated with Large-Amplitude Electron Whistlers," Physics of Fluids 15, 1282-1284 (1972).

Davidson, R. C., Hammer, D. A., Haber, I., and Wagner, C. E., "Nonlinear Development of Electromagnetic Instabilities in Anisotropic Plasmas," Physics of Fluids 15, 317-333 (1972).

Eliasson, B. and Lazar, M., "Nonlinear evolution of the electromagnetic electron-cyclotron instability in bi-Kappa distributed plasma," Physics of Plasmas 22, 062109 (2015).

Gary, S. P. and Feldman, W. C., "A second-order theory for k parallel B sub 0 electromagnetic instabilities," Physics of Fluids 21, 72-80 (1978).

Hellinger, P., Trávníček, P., Kasper, J. C., and Lazarus, A. J.,
"Solar wind proton temperature anisotropy: Linear theory and WIND/SWE observations," GRL 33, L09101 (2006).

Heuer, M. and Marsch, E., "Diffusion plateaus in the velocity distributions of fast solar wind protons," Journal of Geophysical Research (Space Physics) 112, A03102 (2007).

Kennel, C. F. and Petschek, H. E., "Limit on Stably Trapped Particle Fluxes," JGR 71, 1 (1966).

Lazar, M., "The electromagnetic ion-cyclotron instability in biKappa distributed plasmas," AA 547, A94 (2012).

Lazar, M. and Poedts, S., "Instability of the parallel electromagnetic modes in Kappa distributed plasmas - II. Electromagnetic ion-cyclotron modes," MNRAS 437, 641-648 (2014).

Lazar, M., Poedts, S., and Michno, M. J., "Electromagnetic electron whistler-cyclotron instability in bi-Kappa distributed plasmas," AA 554, A64 (2013).

Lazar, M., Poedts, S., and Schlickeiser, R., "Instability of the parallel electromagnetic modes in Kappa distributed plasmas I. Electron whistler-cyclotron modes," MNRAS 410, 663-670 (2011).

Lazar, M., Schlickeiser, R., and Poedts, S., "Suprathermal particle populations in the solar wind and corona," in Exploring the Solar Wind, edited by M. Lazar (InTech, 2012) Chap. 11.

Lazar, M., Schlickeiser, R., and Shukla, P. K., "Cumulative effect of the Weibel-type instabilities in symmetric counterstreaming plasmas with kappa anisotropies," Physics of Plasmas 15, 042103-042103 (2008), arXiv:0802.0570 [physics.plasm-ph].

Lu, Q., Zhou, L., and Wang, S., "Particle-in-cell simulations of whistler waves excited by an electron $\kappa$ distribution in space plasma," Journal of Geophysical Research (Space Physics) 115, A02213 (2010).

Lysak, R. L., "Electrodynamic coupling of the magnetosphere and ionosphere," SSREV 52, 33-87 (1990).

Marsch, E., "On resonant interactions of ions with plasma waves in a reduced quasi-linear theory," Nonlinear Processes in Geophysics 9, 69-74 (2002).

Pierrard, V. and Lazar, M., "Kappa Distributions: Theory and Applications in Space Plasmas," Solar Physics 267, 153-174 (2010), arXiv:1003.3532 [physics.space-ph].

Sagdeev, R. Z. and Shafranov, V. D., "On the Instability of a plasma with an anisotropic distribution of velocities in a magnetic field," JETP 12, 130-132 (1961).

Sarfraz, M., Saeed, S., Yoon, P. H., Abbas, G., and Shah, H. A., "Macroscopic quasi-linear theory of electromagnetic electron cyclotron instability associated with core and halo solar wind electrons," Journal of Geophysical Research (Space Physics) 121, $10(2016)$.

Seough, J. and Yoon, P. H., "Quasilinear theory of anisotropy-beta relations for proton cyclotron and parallel firehose instabilities," Journal of Geophysical Research (Space Physics) 117, A08101 (2012).

Seough, J., Yoon, P. H., and Hwang, J., "Quasilinear theory and particle-in-cell simulation of proton cyclotron instability," Physics of Plasmas 21, 062118 (2014).

Seough, J., Yoon, P. H., Kim, K.-H., and Lee, D.-H., "Solar-Wind Proton Anisotropy Versus Beta Relation," Physical Review Letters 110, 071103 (2013).

Stasiewicz, K., Bellan, P., Chaston, C., Kletzing, C., Lysak, R., Maggs, J., Pokhotelov, O., Seyler, C., Shukla, P., Stenflo, L., Streltsov, A., and Wahlund, J.-E., "Small Scale Alfvénic Structure in the Aurora," SSREV 92, 423-533 (2000).

Štverák, Š., Trávníček, P., Maksimovic, M., Marsch, E., Fazakerley, A. N., and Scime, E. E., "Electron temperature anisotropy constraints in the solar wind," Journal of Geophysical Research: Space Physics 113 (2008).

Summers, D. and Thorne, R. M., "The modified plasma dispersion function," Physics of Fluids B 3, 1835-1847 (1991).

Trakhtengerts, V. Y., "The Mechanism of Generation of Very Low Frequency Electromagnetic Radiation in the Earth's Outer Radiation Belt," Geomagnetism and Aeronomy 3, 365 (1963).

Vedenov, A. A., Velikhov, E. P., and Sagdeev, R. Z., "Special 
Issue: Stability of Plasma," Soviet Physics Uspekhi 4, 332-369 (1961).

Xue, S., Thorne, R. M., and Summers, D., "Electromagnetic ioncyclotron instability in space plasmas," JGR 98, 17475-17484 (1993).

Xue, S., Thorne, R. M., and Summers, D., "Parametric study of electromagnetic ion cyclotron instability in the Earth's magnetosphere," JGR 101, 15467-15474 (1996).

Yoon, P. H. and Seough, J., "Quasilinear theory of anisotropy-beta relation for combined mirror and proton cyclotron instabilities,"
Journal of Geophysical Research (Space Physics) 117, A08102 (2012).

Yoon, P. H., Seough, J. J., Kim, K. H., and Lee, D. H., "Empirical versus exact numerical quasilinear analysis of electromagnetic instabilities driven by temperature anisotropy," Journal of Plasma Physics 78, 47-54 (2012).

Zimbardo, G., Greco, A., Sorriso-Valvo, L., Perri, S., Vörös, Z., Aburjania, G., Chargazia, K., and Alexandrova, O., "Magnetic Turbulence in the Geospace Environment," SSREV 156, 89-134 (2010). 